\title{
RES-Q Register in Bulgaria
}

\author{
S. Andonova ${ }^{1}$, R. Fuchidzhieva ${ }^{1}$ M. Tsalta-Mladenov ${ }^{1}$ \\ ${ }^{1}$ University Hospital "Sveta Marina", Second clinic of Neurology with ICU and Stroke unit, \\ Varna, Bulgaria
}

\section{Background and Aims}

Currently, in Bulgaria there is no official national registry of acute ischemic stroke (AIS) patients who have gone through thrombolytic treatment. Therefore, there is no possibility to perform a national analysis of the impact of different treatments on the outcome of the disease. The aim of our study is to evaluate characteristics from the Res- $Q$ database, related with the quality parameters included in the Res-Q register from all centers in Bulgaria until 2018.

Method

Since there is no national registry of AIS patients, and for the aim of analyzing the results from our research data related to treatment optimization, we have to compare the generalized analysis of the data at the clinic to the database of the remaining centers included in the RES-Q.

Based on the results from the Res-Q register for the period 2016-2018, in the beginning of 2017 the number of registered patients with ischemic stroke was 127 . Out of them, $3(2,36 \%)$ had thrombolysis and there were no registered patients with door-to-needle time under 60 minutes. In the end of 2017 from 495 patients, $58(11,72 \%)$ were treated with thrombolysis. The door-to-needle time was under 60 minutes for $44(75 \%)$ of them and under 45 minutes in $25(43 \%)$ cases.

\section{Conclusion}

There is a significant enhancement in intravenous thrombolysis' results. Adding more centers for stroke treatment to the Res-Q Registry in Bulgaria and the cooperation between those centers are the reasons leading to improved stroke treatment. This proves the necessity of regular and complete data entry procedures to the Registry.

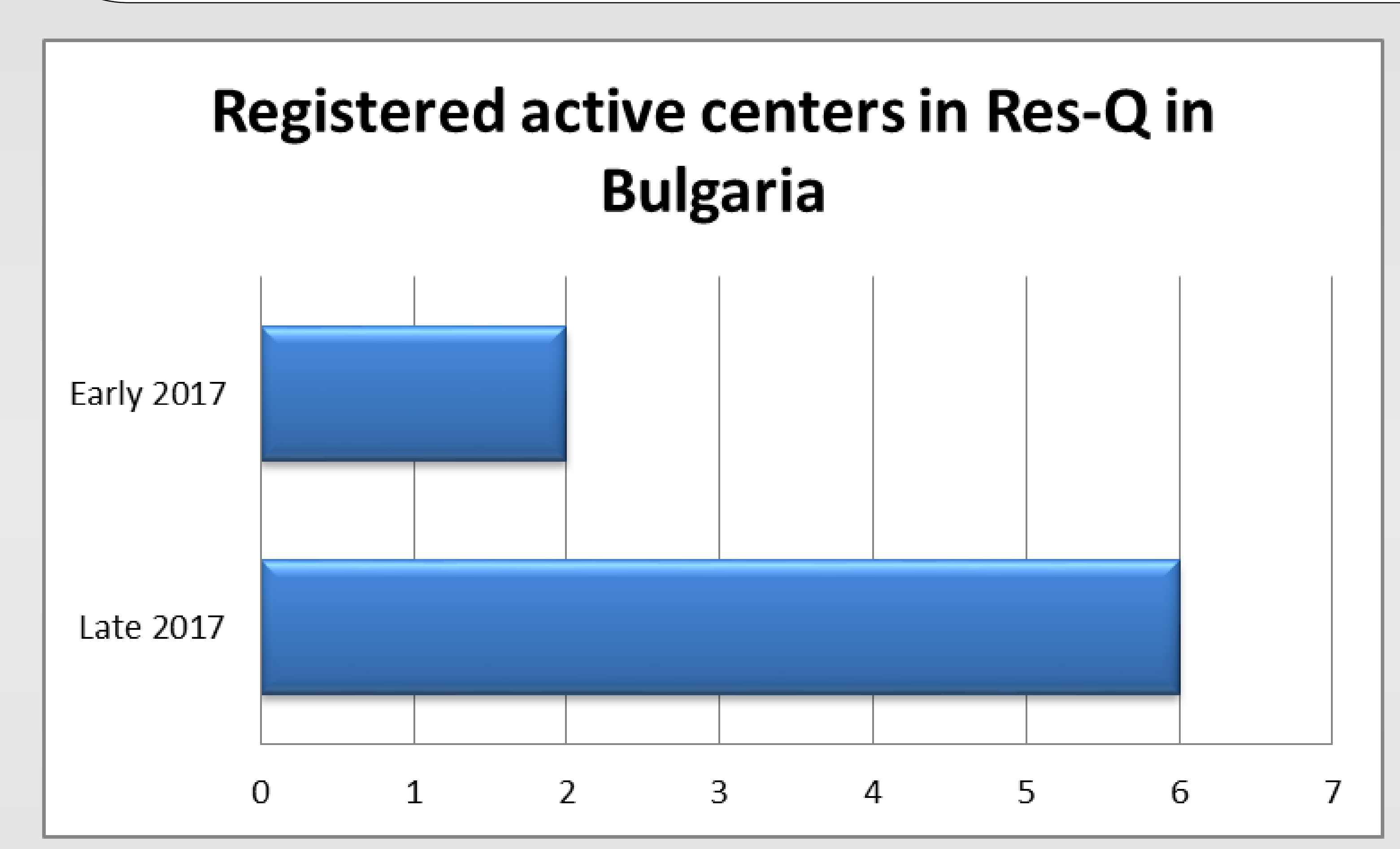

IVT door-to-needle time

From all centers in Bulgaria $\quad$ University Hospital St.Marina Varna

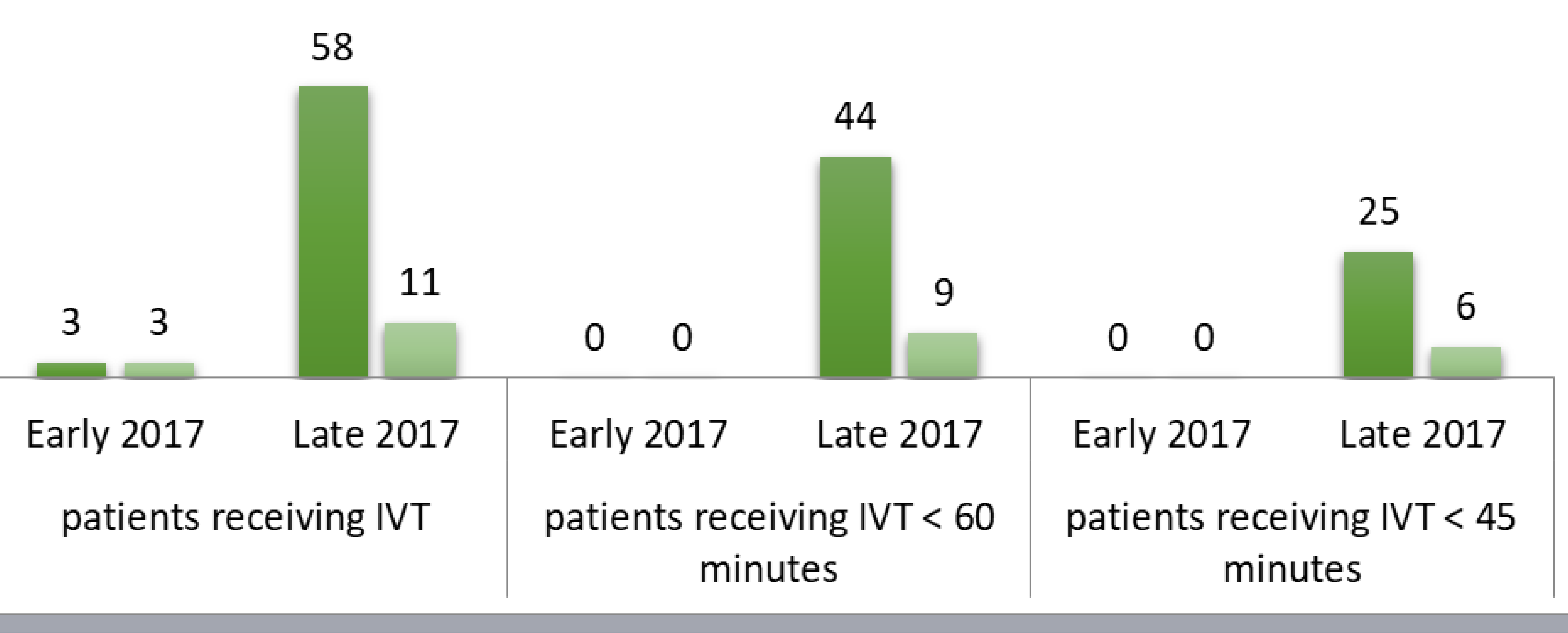

$\%$ patients receiving antiplatelets without AF University Hospital St.Marina Varna

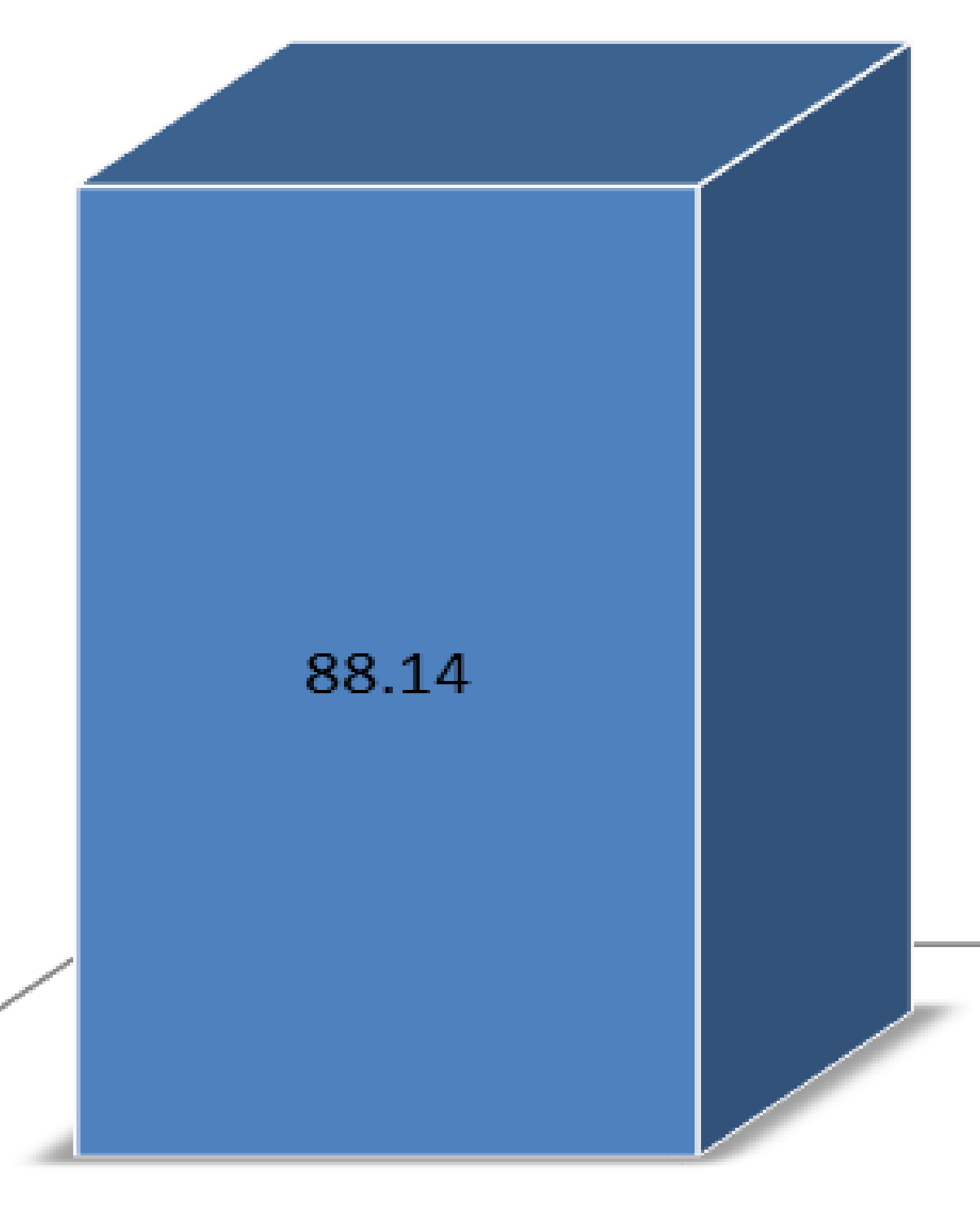

Early 2017
Registered patients with ischemic stroke in early 2017

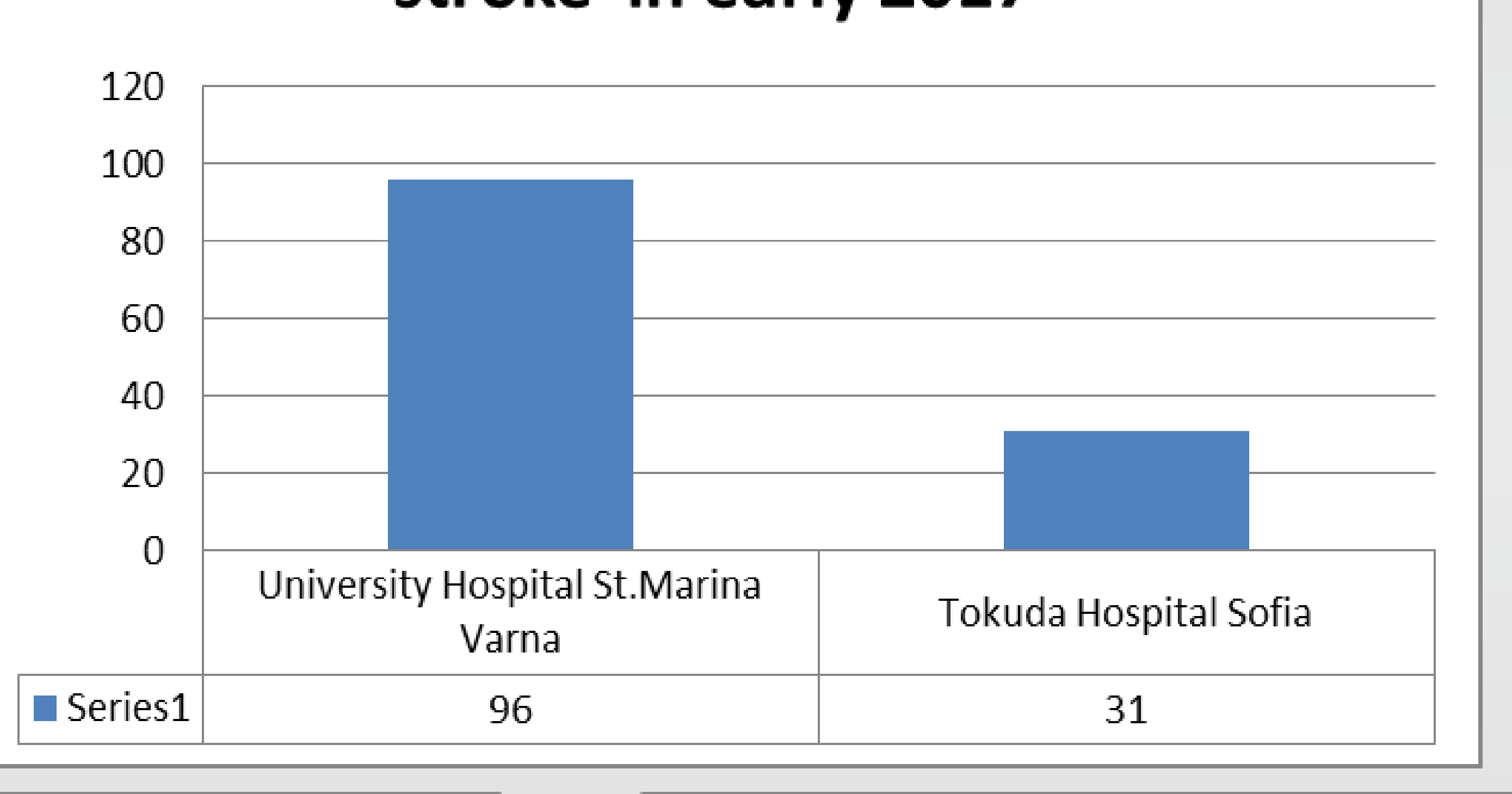

\% patients prescribed anticoagulants with AF University Hospital St.Marina Varna stroke in late 2017
Registered patients with ischemic
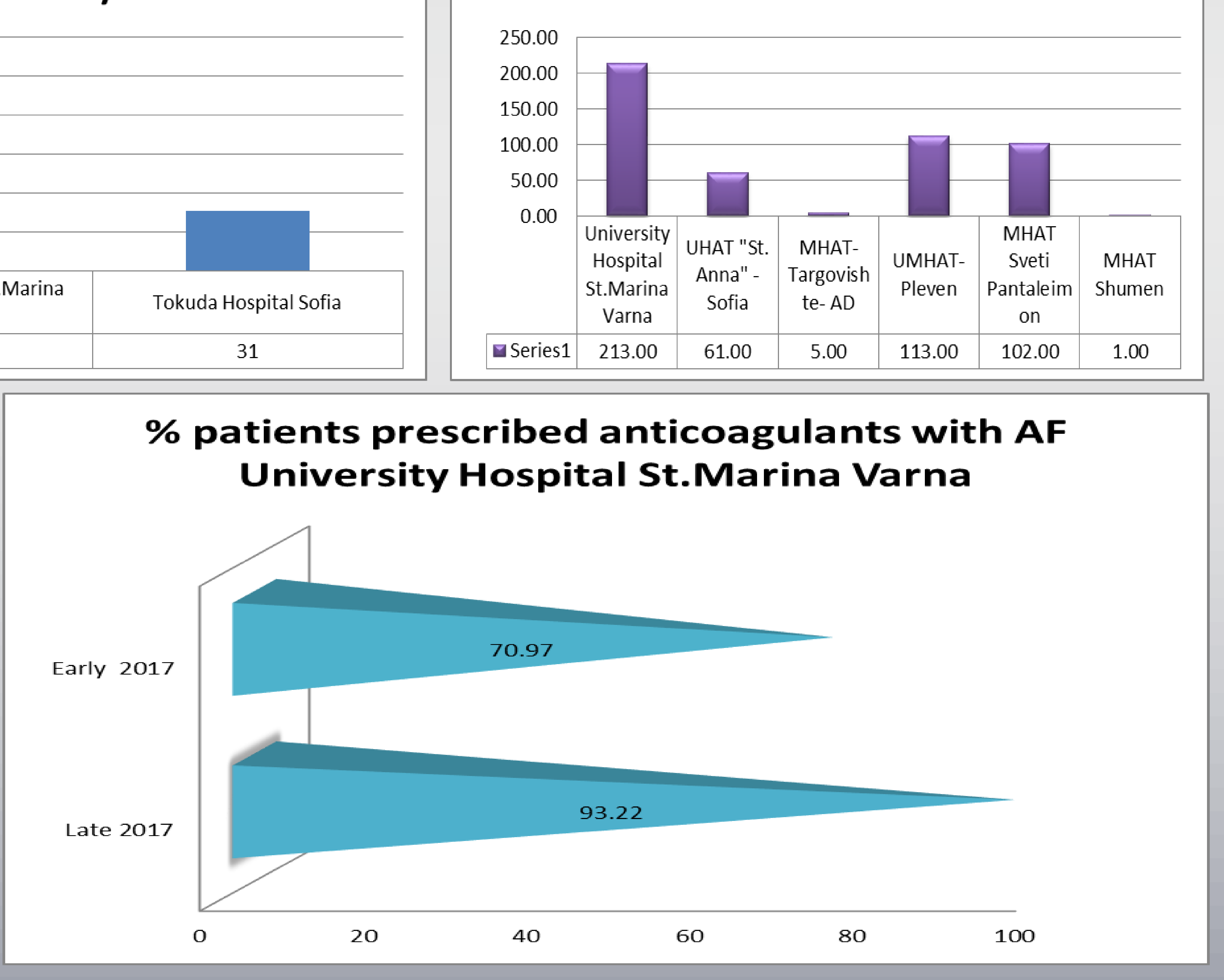

$\%$ patients receiving statins at discharge University Hospital St.Marina Varna

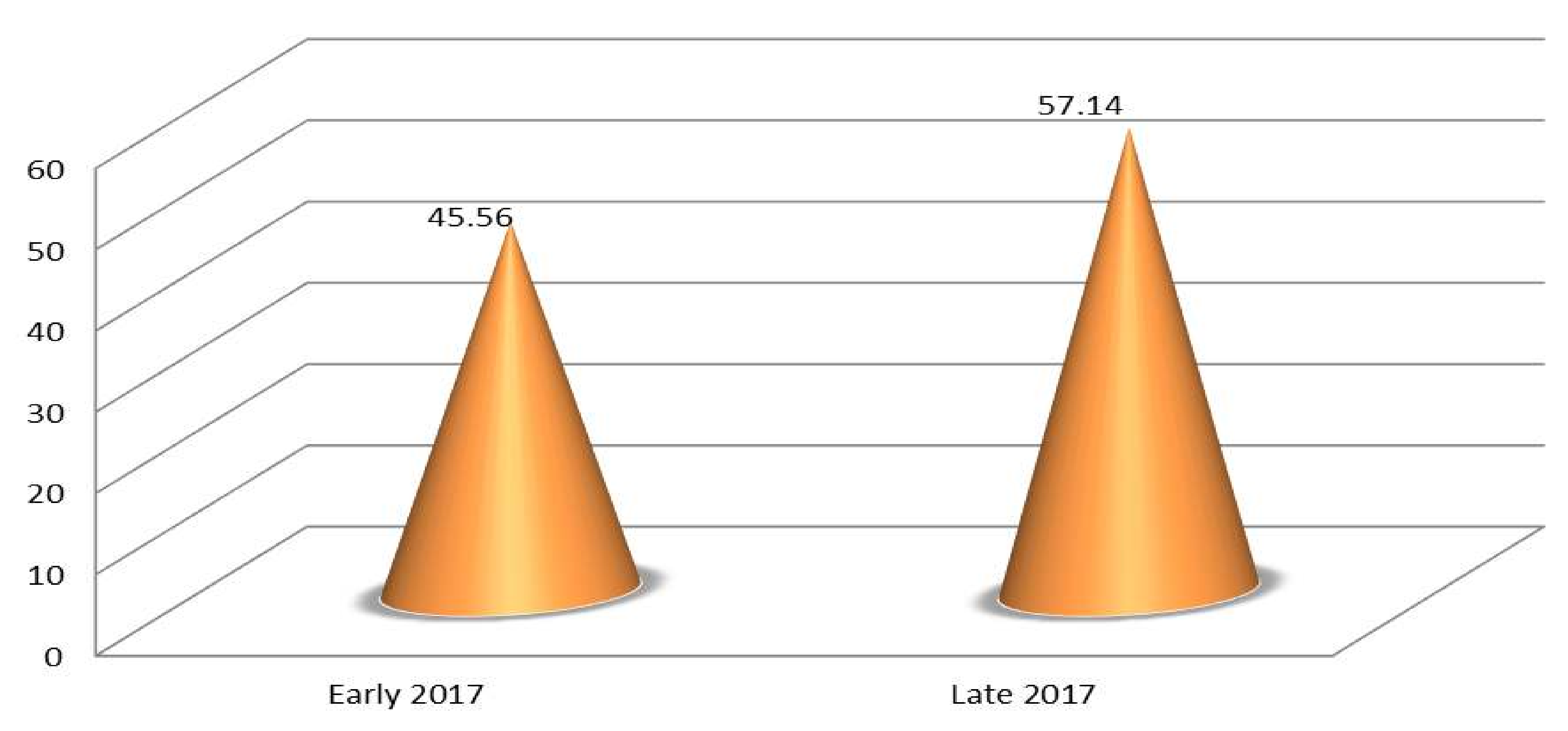

